\title{
The Dynamical Mass of the Young Cluster W3 in NGC 7252: Heavy-Weight Globular Cluster or Ultra-Compact Dwarf Galaxy?
}

\author{
Claudia Maraston, N. Bastian, R. P. Saglia, Markus Kissler-Patig, \\ François Schweizer, Paul Goudfrooij \\ MPE, Giessenbachstraße, 85748 Garching, Germany \\ Universitäts-Sternwarte, Scheinerstraße 1, 81679 München, Germany
}

\begin{abstract}
We have measured the dynamical mass of the highly luminous star cluster W3 in the young merger remnant galaxy NGC 7252. The value is $M_{\mathrm{dyn}}=(8 \pm 2) \times 10^{7} M_{\odot}$, and represents the highest dynamically-confirmed mass for an extra-galactic star cluster so far. The dynamical mass is in excellent agreement with the luminous mass (Maraston et al. 2001). This results from the use of stellar population models that include correctly the brightest AGB stellar phase, dominant in young stellar populations. To classify W3, we employ the fundamental plane of stellar systems (Bender, Burstein \& Faber 1992), for the first time in these kinds of studies. We find that W3 lies far from typical Milky Way globular clusters, but it is also far from the heavyweights $\omega$ Cen in the Milky Way and G1 in M31, because it is too extended for its mass, and from dwarf elliptical galaxies because it is much more compact for its mass. Instead W3 lies close to the ultra-compact Fornax objects (Drinkwater et al. 2003) and to the compact elliptical M32, possibly shedding light on the still mysterious nature of these objects. A previously deserted region of the fundamental plane starts to be populated.
\end{abstract}

Extreme star bursts seen in galaxy mergers are able to produce star clusters with masses up to $10^{7} M_{\odot}$, that are suggested to evolve into galactic globular clusters (GC). However some objects seem to escape such notice, because their luminosity-estimated masses are much larger than those of the most massive GCs. The most extreme case is a star cluster in the young ( $t \lesssim 1 \mathrm{Gyr}$ ) merger remnant galaxy NGC 7252, for which the luminous mass was found to be nearly $10^{8} M_{\odot}$ (Schweizer \& Seitzer 1998; Maraston et al. 2001). Therefore it is important to check the dynamical mass (Maraston et al. 2004). To this aim, the internal velocity dispersion of the object has been measured from a highresolution, high $S / N$ optical spectrum obtained with UVES/VLT, by means of the FCQ method (Bender 1990). We use a composite stellar template in which the luminosity contributions of the individual stars are those of the stellar population model that matches the spectra and colors of W3 $(t \sim 300 \mathrm{Myr}$, $Z \sim 0.5 Z_{\odot},[\alpha / F e] \sim 0$; Maraston et al. 2001). Our result is the astonishingly high velocity dispersion $\sigma=45 \pm 5 \mathrm{~km} / \mathrm{s}$, that combined with the large cluster size $R_{\text {eff }}=17.5 \pm 1.8 \mathrm{pc}$, translates into a dynamical virial mass for W3 of $(8 \pm 2) \times 10^{7} M_{\odot}$. In order to classify this object, we use the fundamental plane (Figure 1), on which we place the new UCDGs and the massive Local Group star clusters $\omega$ Cen and G1. W3 lies far from the average line of galactic GCs and from $\omega$ Cen and G1. Instead, a connection might exist with the UCDGs and with the compact elliptical M32. 


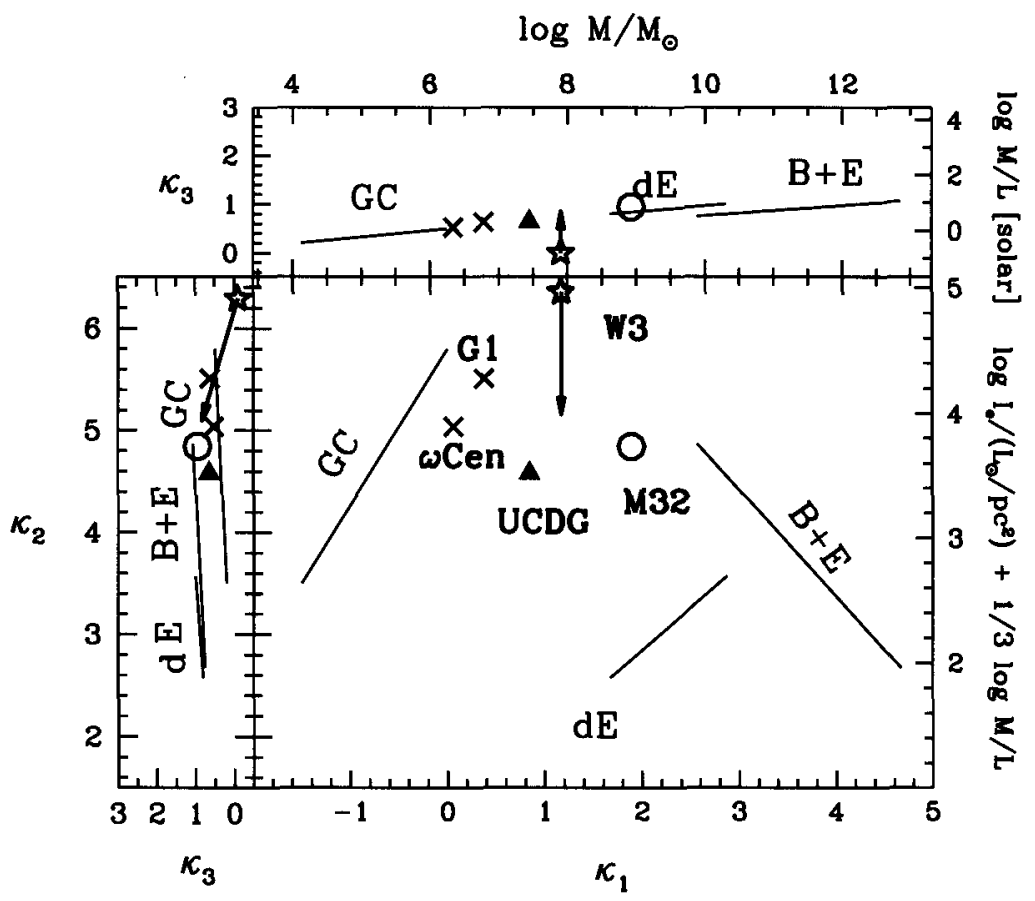

Figure 1. The fundamental plane of stellar systems - bulges plus ellipticals $\mathrm{B}+\mathrm{E}$, dwarf ellipticals dE, globular clusters GC (Burstein et al. 1997) in the version containing massive star clusters and Ultra Compact Dwarf Galaxies (UCDGs) (Maraston et al. 2003). $\kappa_{1}$ is the mass of an object, while $\kappa_{2}$ can be interpreted as a concentration factor in the sense that objects with higher values of $\kappa_{2}$ are more concentrated for a given mass. $\kappa_{3}$ is the mass-to-light ratio. The arrow from W3 indicates its position when faded to $10 \mathrm{Gyr}$ due to stellar evolution. Note that dynamical mass losses are not taken into account (see discussion in Maraston et al. 2003).

\section{References}

Bender, R. 1990, A\&A, 229, 441

Bender, R., Burstein, D., Faber, S.M. 1992, ApJ, 399, 462

Burstein, D., Bender, R., Faber, S.M., Nolthenius, R. 1997, AJ, 114, 1365

Drinkwater, M.J., et al., 2003, Nature, 423, 519

Maraston C., Bastian, N., Saglia, R. P., Kissler-Patig M., Schweizer, F., Goudfrooij, P. 2004, A\&A, 416, 467

Maraston C., Kissler-Patig, M., Brodie, J., et al. 2001, A\&A, 400, 823

Schweizer, F., Seitzer, P. 1998, AJ, 116, 2206 